\section{REVISTA}

Actualidades Investigativas en Educación

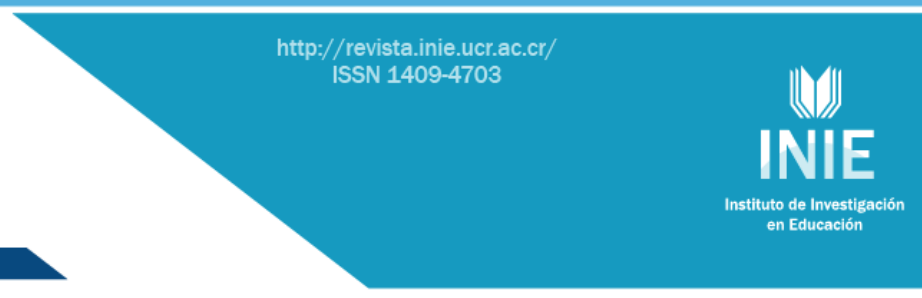

\title{
EL PAPEL DEL DOCENTE EN LA EDUCACIÓN PARA LA SEXUALIDAD: ALGUNAS REFLEXIONES EN EL PROCESO EDUCATIVO ESCOLAR
}

THE TEACHER'S ROLE IN SEXUALITY EDUCATION: THE THOUGHTS IN THE SCHOLAR EDUCATIVE PROCESS

\section{Volumen 15, Número 3}

Setiembre - Diciembre

pp. $1-15$

DOI: http://dx.doi.org/10.15517/aie.v15i3.20335

Yanine González Gómez

Revista indizada en REDALYC, $\underline{\text { SCIELO }}$

Revista distribuida en las bases de datos:

CATÁLOGO DE LATINDEX, IRESIE, CLASE, DIALNET, DOAJ, E-REVIST@S, SHERPA/ROMEO, QUALIS, MIAR

Revista registrada en los directorios:

ULRICH'S, REDIE, RINACE, OEI, MAESTROTECA, PREAL, CLACSO

Los contenidos de este artículo están bajo una licencia Creative Commons

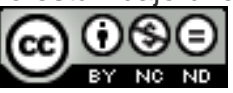




\title{
EL PAPEL DEL DOCENTE EN LA EDUCACIÓN PARA LA SEXUALIDAD: ALGUNAS REFLEXIONES EN EL PROCESO EDUCATIVO ESCOLAR
}

\author{
THE TEACHER'S ROLE IN SEXUALITY EDUCATION: THE THOUGHTS IN THE SCHOLAR \\ EDUCATIVE PROCESS
}

\section{Yanine González Gómez}

\begin{abstract}
Resumen: Este ensayo presenta algunas posturas teóricas relacionadas con la educación para la sexualidad en las escuelas y el papel de las y los docentes en este proceso formativo. Se parte, de una revisión analítica de la literatura en el marco de una investigación doctoral realizada entre los años 2011 al 2014, que explora las aspiraciones a una mejor calidad de vida en mujeres con edades entre los 12 y 16 años con experiencia de violencia doméstica. Se reflexiona acerca del rol del personal docente, del impacto de los proyectos de educación para la sexualidad en la población educativa y se discute sobre la forma en que esta población gestiona sus miedos, estereotipos, desconocimientos y mitos acerca de la sexualidad cuando deben asumir la educación de sus estudiantes, con quienes tienen diferencias generacionales y culturales. Se concluye que la sexualidad no debe verse como un escenario de disputa y exclusión en las escuelas, sino como un espacio de aprendizaje y creación, que no es estático y no solo la población adolescente y joven debe aprender de esta, brindando un ambiente para pensarla, repensarla y de ser posible, reaprenderla a lo largo de la vida; por último se menciona la perspectiva intercultural como alternativa para abordar la educación para la sexualidad en las instituciones educativas, dado que posibilita el encuentro de diversas formas de ser y estar en el mundo, ampliando la construcción de las identidades, la reflexión y el compromiso político, frente a las diversidades.
\end{abstract}

Palabras clave: EDUCACIÓN PARA LA SEXUALIDAD, GÉNERO, DOCENTES, INTERCULTURALIDAD, ADOLESCENCIA, COLOMBIA.

\begin{abstract}
This paper presents some theoretical positions related with the sexuality for education in schools and the role of teachers and in the formative process. It is part of an analytical review of the literature in the context of a doctoral research between 2011 and 2014, with the tarjet of explores the aspirations for a better quality of life in women aged between 12 and 16 years with experience in domestic violence. We reflect on the role of teachers, the impact of projects on education for the sexuality in the students and discusses how this teachers manages their fears, stereotypes, unknowns and myths about sexuality when they should take education of their students, who have generational and cultural differences. We conclude that sexuality should not be seen as a stage of dispute and exclusion in schools, but as a space for learning and creation, which is not static, not just adolescents and young should learn from this, providing an environment to think it, rethink it and, if possible, learn again throughout life; finally the intercultural perspective as an alternative to assume sexuality for education in educational institutions, because it permits the communication of different ways to be and to be in the world, expanding the construction of identities, reflection and political commitment versus diversity
\end{abstract}

Key words: SEXUALITY FOR EDUCATION, GENDER, TEACHERS, INTERCULTURALITY, ADOLESCENCE, COLOMBIA

\footnotetext{
1 Docente de la Universidad Antonio Nariño, BogotáColombia. Psicóloga, Magister en Investigación Social Interdisciplinaria.
}

Dirección electrónica: yanine.gonzalez@uan.edu.co

Ensayo recibido: 15 de octubre, 2014

Enviado a corrección: 3 de marzo, 2015

Aprobado: 13 de julio, 2015 


\section{Introducción}

Los elementos de partida para este escrito se pueden ubicar en la elección de tres interrogantes (entre otros) planteados por Morgade, Baez, Zattara y Díaz (2011, p.23) referidos a las pedagogías de género y la educación sexual, en los que se cuestionan frente a la educación sexual: ¿Qué se enseña?, ¿Quién lo enseña? ¿Qué sabe quién "enseña"?,; a estos interrogantes propuestos por las autoras podemos sumar ¿Cómo gestionan las y los docentes, miedos, estereotipos, desconocimientos y mitos acerca de la sexualidad?.

Para Morgade (2011b), la presencia en las ultimas décadas de diversas situaciones de orden social, político y económico en América Latina posibilitaron la apertura de escenarios en los que se planteó el estudio y el análisis de temáticas referidas a la sexualidad en los contextos escolares. Por ejemplo, las preocupaciones de salud pública relacionadas con la aparición de las infecciones de transmisión sexual, el VIH-SIDA y los embarazos en adolescentes generaron empezar a hablar de ellos en la escuela de una manera preventiva; esto conllevó en muchos contextos a la biologización de la educación sexual con su consecuente medicalización como opción formativa y a la construcción de políticas públicas al respecto.

Para Morgade y et al, (2011a) las luchas sociales, el cambio en los paradigmas educativos y la aparición, visibilización o transformación de algunas prácticas culturales exigieron cambios en la manera en que era y es pensada la sexualidad en los escenarios educativos. Solo por mencionar algunos están, los movimientos sociosexuales, de mujeres, de género, gays y lesbianas, las teorías feministas y de género, la pedagogía crítica, los movimientos queer, entre otros evidenciaron los diferentes modos de subordinación y discriminación que se justificaban en las prácticas sexuales de hombres y mujeres.

Para Lopes Louro (2000) las transformaciones sociales mencionadas anteriormente, daban lugar a nuevas formas de relación que en algunos sectores podrían aparecer como perturbadoras, pero siéndolo o no, movilizaron cambios en ellos que parecían inamovibles, dando lugar a nuevas identidades de género y sexuales, nuevas formas de aparecer en el mundo, de nacer y crecer en él, de amar, incluso de morir. Afirma la autora:

Tales transformaciones constituyen nuevas formas de existencia para todos, incluso para aquellos que, aparentemente, no las experimentan de modo directo. Ellas permiten nuevas soluciones a indagaciones que surgen $\mathrm{y}$, obviamente, provocan nuevas y desafiantes preguntas. Tal vez sea posible, trazar algunos puntos en común para sustentar las respuestas. El primer punto se refiere a la comprensión de que la 
sexualidad no es sólo un asunto personal, sino social y político. El segundo, al hecho de que la sexualidad es "aprendida" o mejor, se construye durante toda la vida, en muchos sentidos, para todos los sujetos ${ }^{2}$ (p. 5)

En tal sentido, el presente ensayo busca reflexionar acerca del lugar de las y los docentes que, por ejemplo, en el caso colombiano, asumen por asignación directa, como imposición, la "educación sexual" de las y los jóvenes de las instituciones educativas. Se reflexiona acerca del rol del personal docente, del impacto de los proyectos de educación para la sexualidad en la población educativa y se discute sobre la forma en que esta población gestiona sus miedos, estereotipos, desconocimientos y mitos acerca de la sexualidad cuando deben asumir la educación de sus estudiantes, con quienes tienen diferencias generacionales y culturales.

\section{Educación para la sexualidad en Perspectiva}

En Colombia la educación sexual ha sido una cuestión de interés desde hace algo más de tres décadas, las políticas que centran su mirada en la educación sexual y reproductiva se fundamentan inicialmente en los compromisos que se han adquirido como país en el marco de conferencias internacionales pero además en una serie de leyes internas; así por ejemplo, en el marco internacional se identifica la Declaración Mundial sobre la Educación para Todos que tuvo lugar en marzo de 1990 en Tailandia, la Conferencia Internacional sobre la Población y el Desarrollo adelantada en 1994 en el Cairo.

En el ámbito nacional se identifica en primera instancia la Constitución Política de 1991, Artículo 42; los Derechos Sexuales y Reproductivos que se encuentran amparados a su vez por un marco internacional frente a los derechos humanos y por las Naciones Unidas; La Ley General de Educación 0115 de 1994 y la Resolución 3353 de 1993 en la cual el Ministerio de Educación Nacional (MEN) le otorgó el carácter de obligatoriedad mediante la fundamentación del Proyecto Nacional de Educación Sexual (PNES), desde entonces se han desarrollado programas, proyectos y políticas encaminadas hacia la promoción de prácticas sexuales responsables; el decreto 1860 de 1994 en el que se reglamenta parcialmente la Ley 115 de 1994 y la Política Nacional de Salud Sexual y Reproductiva de 2003, que como objetivo central busca mejorar la Salud Sexual y Reproductiva y promover el ejercicio de los Derechos Sexuales y Reproductivos de toda la población con especial énfasis en la

2 La traducción es mía 
reducción de los factores de vulnerabilidad y los comportamientos de riesgo, el estímulo de los factores protectores y la atención a grupos con necesidades específicas.

Lo anterior da cuenta de una trayectoria importante en el país en busca de mejorar las condiciones de hombres, mujeres, niñas y niños en los temas referidos a la sexualidad. No obstante, los resultados a la fecha no evidencian mayores cambios; por ejemplo, la última Encuesta Nacional en Demografía y Salud llevada a cabo por la Asociación Probienestar de la Familia Colombiana (Profamilia, 2010) revela que aún existe desconocimiento de las mujeres sobre prácticas seguras para evitar el contagio del VIH/SIDA y que, incluso, entre quienes han recibido información, casi la mitad vincula el contagio con la homosexualidad. Asimismo, se encontró que el nivel educativo y los índices de pobreza se siguen relacionando de manera inversamente proporcional con la edad mediana de inicio de las relaciones sexuales.

De la misma manera, un estudio reciente sobre la salud mental en adolescentes colombianos cuestiona los programas de salud en materia de educación en sexualidad, dado que por ejemplo entre aquellas adolescentes que reportan tener una vida sexual activa, el $33,2 \%$ siempre tiene relaciones con condón, pero la misma proporción nunca cuenta con dicha protección, razón por la que quedan expuestas a embarazos no deseados y abortos, fuera del alto riesgo de enfermedades de transmisión sexual (Torres, Berbesi, Bareño y Montoya, 2010).

De acuerdo a Morgade

...es recurrente en los diferentes estudios la constatación de que cuando se han abordado en forma explícita temáticas vinculadas con las sexualidades, existe una distancia entre la transmisión de información y su apropiación transformativa por parte de las/los jóvenes y adultos/as jóvenes. Una distancia que ha llevado a evaluar como infructuosos muchos esfuerzos por llegar a la juventud desde las campañas de prevención. (2011, p. 15)

Al respecto, estudios financiados por la Secretaría de Educación Distrital de Bogotá (SED) y llevados a cabo por la Fundación GAMMA IDEAR en el año 2006 demuestran que en los colegios públicos de Bogotá, la mayoría de los proyectos en educación sexual y prevención no cuentan con objetivos claros ni con indicadores que informen sobre el avance obtenido hacia su cumplimiento; se evidencia una influencia mayor de los pares y medios de comunicación en los adolescentes que del ámbito escolar. Según los mismos docentes, 
existe un trabajo desarticulado entre áreas y una gran cantidad de actividades y talleres que en últimas se convierten en una carga para ellos y redundan en un activismo poco funcional. En este contexto, los proyectos masivamente enfocados funcionan a nivel informativo y no actitudinal, esto vinculado al hecho de que quienes los diseñan son adultos que se ubican lejos de las necesidades sentidas de los jóvenes, desde una postura reacia al cambio y que aún no logran la aceptación de la diferencia. En este punto se menciona un fuerte cuestionamiento a los currículos, metodologías y al incumplimiento y/o debilidad en la educación universitaria en las temáticas y destrezas requeridas para la educación sexual.

En la medida en que los materiales de la educación sean elegidos por su susceptibilidad a la transformación imaginativa y sean presentados de modo que inviten a la negociación y la especulación, la educación llega a formar parte de lo que Bruner (1994) denomina "elaboración de cultura"; sin embargo, se encuentra con frecuencia que las condiciones sobre las que se desarrollan los procesos de aprendizaje en la escuela están mediados por la poca participación activa de las y los estudiantes en la elección de los currículos y metodologías de evaluación; y en este contexto, el tema de la educación en sexualidad no es la excepción.

Es frecuente encontrar en las instituciones educativas colombianas que los programas de educación en sexualidad sean de carácter informativo en lugar de formativo, priorizando el enfoque sexológico - biomédico y de prevención de riesgos, (Rosales y Flores, 2009); a pesar de las falencias de estos proyectos, la política de obligatoriedad ha permitido que los programas de educación sexual existentes tengan una mayor cobertura que en el pasado. No obstante, el principal aspecto que podría explicar la ineficiencia de dichos programas es el hecho de que las instituciones educativas han dejado de lado las verdaderas necesidades de aprendizaje de la población estudiantil (Valencia y Solera, 2011). Es claro el sesgo con el que se tratan los aspectos relacionados con la sexualidad humana, estos son reducidos a una esfera netamente informativa elaborada desde lo que los adultos consideran prioridad, a partir de los riesgos a los que se enfrenta la juventud.

El hecho de que los programas brinden información sobre el cuerpo y la sexualidad no es un error, por el contrario, es un paso fundamental en el proceso de formación de individuos conscientes y responsables, el cual puede orientar el quehacer de sus prácticas. El problema surge cuando dichos programas no toman en cuenta el componente afectivo e interrelacional que implica la sexualidad humana, viéndola sólo desde una perspectiva de pareja heterosexual, culturalmente aceptada, sin incluir prácticas y otras sexualidades que 
cada día se visibilizan más. Lo anterior se refleja en una investigación titulada "Necesidades de aprendizaje para la sexualidad de jóvenes entre 10 y 19 años en el Caribe colombiano" (Valencia y Solera, 2011).

En dicho estudio se explicita cómo las necesidades de aprendizajes para la formación de la sexualidad en los adolescentes están relacionadas con el ser, y se orientan hacia el desarrollo de tópicos afectivos. Los jóvenes desean ser formados como personas con sentimientos, emociones, valores y no simplemente como receptores del conocimiento elaborado y ajustado a las necesidades de los adultos. Las necesidades o "estrategias de potenciación del amor" se convierten en un camino de transformación de la misma sociedad, en virtud de que los jóvenes desean ser ellos mismos, olvidarse de los factores externos y reencontrarse con su identidad (Valencia y Solera, 2011, p. 122).

Al respecto Muñoz (2012) sostiene:

Los jóvenes están saturados de que se les hable de temas como la anticoncepción, las ITS (especialmente el VIH/SIDA) y el embarazo no deseado. Al parecer aspectos más volitivos del encuentro sexual, así como la reflexión sobre condiciones culturales que median en el mismo tendrían mayor recepción. La discusión sobre paradigmas como la pareja y la monogamia, sobre los valores afectivos asociados al sexo y su tensión con la búsqueda exclusiva del placer, así como sobre las diferencias de géneros han demostrado tener acogida entre jóvenes de distintas ciudades y contextos, siempre y cuando no perciban una imposición del mundo valorativo de los adultos. (pp. 18-19)

Para Morgade (2006) en la escuela, la educación en sexualidad con perspectiva "médica" se basa en tres aspectos de la prevención:

a) Prevención centrada en el cuerpo humano, el cual se puede embarazar, enfermar, alcoholizarse, hacerse adicto, suicidarse. Es necesario, dice la autora, "contener" un cuerpo que se puede desordenar siendo primordial humanizarlo, disciplinarlo.

b) Prevención impartida por voces especialistas, voces autorizadas la mayoría de las veces de profesionales en medicina o psicología a quienes se recurre fuera de la institución escolar; al interior de esta son generalmente los profesores de biología quienes educan (previenen) en sexualidad. Las y los expertos detentan un saber legitimado, que desautoriza, silencia y/o aísla los saberes de otros agentes educativos. 
c) Por último, presenta la autora la prevención como algo que compete a mujeres y a heterosexuales. Así, la prevención circula entorno a los embarazos tempranos, a las infecciones de transmisión sexual, al abuso sexual, es decir, se presenta la sexualidad como riesgosa y patológica.

Es claro que cuando se trata acerca de temas de sexualidad las estrategias basadas en la información no inciden en el comportamiento de quienes la reciben, menos, si dicha información se nutre de estereotipos, mitos o desinformaciones que contravienen los derechos de aquellos y aquellas que son percibidos como lo "Otro". Las diversas situaciones sociales que atraviesan las y los jóvenes en las cuales su vida corre peligro o la pierden implican mucho más que disponer de información o, incluso, de "métodos" de anticoncepción o contra el contagio de infecciones (Morgade, 2011b, p. 16).

Enfatiza Muñoz (2012) la necesidad de implementar nuevas estrategias que superen las limitaciones de los actuales procesos pedagógicos, dentro de estas incluye el desarrollo afectivo en el ambiente escolar, el trabajo grupal a partir de la confianza y la amistad, así como el desarrollo de programas pedagógicos que incorporen la resolución positiva de conflictos y la generación de espacios reflexivos, entre otros. Además de lo anterior, el mismo autor resalta las necesidades de incluir elementos como la pedagogía del tiempo libre, la democracia y el respeto por las diferencias. Consecuentemente, se torna necesario preguntarse si dichas dificultades en materia de formación en sexualidades, además de la desarticulación y escasa planeación conjunta, no responde a un componente individual de aquellas y aquellos docentes encargados de formar alos niños, a las niñas y a las y los jóvenes.

En este sentido, Bellhooks, citada por Morgade (2013) considera que "las profesoras" entran en la clase decididas a anular el cuerpo, aceptando el supuesto de que la pasión no tiene lugar en el aula; para Morgade, quienes asumen el reto de educar en sexaulidades, deben en primera instancia transitar por rutas de desaprendizajes, borramientos y negociaciones frente a las comprensiones que se poseen de la sexualidad como mandato y/o prohibición.

Para Caricote (2008), citando a Montiel, los docentes traen consigo procesos de formación marcados por la represión de la sexualidad, lo que les genera un sentir impreciso y en ocasiones negativo frente a esta. Las y los docentes que deben, en la actualidad, encargarse de la educación sexual en las escuelas han pasado por sistemas educativos en 
los cuales la experiencia de la sexualidad se ha relacionado con el temor, con la prohibición, con la advertencia sobre los peligros que ella comporta, de tal manera que en sus escenarios laborales y, por qué no pensarlo, familiares, reproducen el silencio como estrategia frente a la experiencia de la sexualidad.

Así, Caricote (2008) advierte:

... se entiende que los mensajes positivos o negativos que reciben las/los adolescentes acerca de la sexualidad están marcados por la historia de represión de la sexualidad no solo de los padres/madres sino también por los docentes y así se le asigna a estas conductas sexuales de los niños y adolescentes, una valoración moral cuando se dice que están haciendo "algo malo" o que "sabe mucho", si se toca su cuerpo o pregunta sobre su sexualidad (pp. 24-25)

De acuerdo con Heller (1991) cada ser humano hace su aparición en la cotidianidad con todo su acervo personal, dicho acervo es diverso pero a la vez especifico, está enmarcado en la historia y se produce en el entramado de relaciones sociales que establece. Cuando llegamos al mundo, éste no es nuevo, ya estaba y diariamente intentamos participar y permanecer en él:

Todas las capacidades fundamentales, los afectos y los modos de comportamiento fundamentales con los cuales trasciendo mi ambiente y que yo remito al mundo "entero" alcanzable por mí y que yo objetivo en este mundo, en realidad yo me los he apropiado en el curso de la vida cotidiana. (p. 25)

Las interacciones en la vida cotidiana van construyendo en los seres humanos un sistema de normas y posibilidades de cómo ser y actuar en el mundo, cuando los sujetos encuentran inconsistencias en su marco normativo aparecen los prejuicios que, según Ferreria (2012), son definidos por Agnes Heller como el resultado de las reflexiones que hacen los sujetos de sus experiencias enmarcadas en un contexto esencialmente histórico. Aunque dentro de las características principales de los prejuicios están las motivaciones individuales, éstos, los prejuicios no se pueden entender en la individualidad, por el contrario, provienen de la asimilación de una huella ideológica, son por lo tanto individuales y/o compartidos. Lo que posibilita la permanencia de un prejuicio es que él satisface las motivaciones particulares de un sujeto o de un grupo y les protege de los conflictos, de manera pasiva los sujetos se apropian de los pensamientos preestablecidos, por lo que sus 
decisiones y actuaciones pierden el carácter de individualidad. Como consecuencia, cada hombre y mujer tienen prejuicios pero, al mismo tiempo, poseen la responsabilidad individual de la elección relativamente libre por la apropiación de una falsa conciencia.

Para el antropólogo Eduardo de Aquino (1999), en los contextos educativos los prejuicios se relacionan con la dificultad para aceptar la diferencia física, social, étnica, religiosa, sexual, entre otras. Son prejuicios cristalizados que permanecen en el tiempo y no se cuestiona su presencia, con lo cual generan una discriminación negativa, derivando en ocasiones en diversos tipos de violencias.

Se hace necesario por tanto, desde este intento comprensivo, en humanizar el rol de las profesoras y profesores, reconociendo sus miedos, desconocimientos, inseguridades y prejuicios frente a los cuerpos y a las sexualidades también las rupturas y desplazamientos que han hecho frente a los aprendizajes represivos y medicalizados que frente a la sexualidad han recibido a lo largo de la vida. Para Flores (2007), la docencia es un trabajo altamente feminizado, esto hace necesario que las mujeres dedicadas a ella realicen una reflexión seria sobre su propia sexualidad, pensándose como madre/educadora y dando un lugar de reconocimiento a la sexualidad presente en la vida de sus estudiantes. Esta autora sugiere a modo de reflexión preguntas como las siguientes: ¿qué estoy dispuesta a escuchar?, ¿cuál es el punto en que me resulta intolerable pensar y hablar de sexualidad?, ¿qué sucede con aquellas maestras cuyas prácticas sexuales son socialmente estigmatizadas, impugnadas por el sistema heteronormativo?. Estos procesos deliberativos posibilitan relaciones honestas, en las que el lugar del o de la docente más que impartir información, acerque a la co-construcción de saberes validando los aprendizajes previos, dando un lugar a la experiencia.

Para Elizalde (2013), los distanciamientos que se pudieran presentar en el acto pedagógico en el marco de la educación en sexualidades, entre las y los diversos actores intervinientes, relacionadas con los prejuicios, aprendizaje, o brechas generacionales, se pueden negociar desde una dimensión intercultural, que en palabras de la autora es "la puesta en acto de un campo de interlocución. Es decir, como un marco de inscripción de múltiples procesos de intersección"; la "interculturalidad" refiere "a las fronteras entre marcos de significación o entre regímenes de articulación de significados, que enuncian también los sentidos de las identificaciones, las categorías y dimensiones identitarias, los sentidos de pertenencia" (Caggiano, 2005, p. 32; citado por Elizalde, 2013). La perspectiva intercultural posibilita en el contexto educativo el encuentro de diversas formas de ser y estar en el 
mundo, ampliando la construcción de las identidades, la reflexión y el compromiso político, frente a las diversidades.

En esta misma línea Leiva Olivencia (2010), expresa que la educación intercultural abre caminos a una educación en la que haya espacio para todas y todos; una educación inclusiva en la que el encuentro con las diferencias culturales posibilite la riqueza de aprendizajes, otorgando igualdad de oportunidad de acuerdo a la diversidad de géneros, etnias y cultos, roles y generaciones que al interior de la escuela convergen, lo anterior abre nuevos horizontes de acción para las y los docentes, quienes a juicio del autor son de vital importancia para configurar estos procesos de formación intercultural. Así, para Elizalde, el lugar de las y los docentes en la escuela más que transmisores de conocimiento se debe distinguir por la de intelectuales con compromiso social que propendan por la construcción de relaciones sociales democráticas, de respeto y ejercicio de los derechos humanos y sexuales; es claro que este no es un camino de fácil recorrido, pero lo plantea como un reto que necesita ser reflexionado desde la investigación y las prácticas educativa y política (2013).

Es indispensable que cada docente y, de manera particular, cada institución educativa, analice los valores que en su currículo contempla, cómo entiende la escuela el respeto, la familia, la convivencia, la sexualidad, la orientación sexual, cuál es la participación de la familia, de los estudiantes y de los docentes. "...La escuela puede ser un lugar donde se reproducen las desigualdades culturales o un espacio donde tienen lugar encuentros interculturales" (Martínez; 2012. p. 27).

Frente a esto Morgade señala:

Subrayar el valor de la escuela, no como una pretensión voluntarista sino a partir de la constatación de que en su seno se dan procesos de disputa, situaciones de ejercicio de la autonomía que no se dan en otros ámbitos, episodios de contacto respetuoso entre culturas y subjetividades diversas, e inclusive, momentos de "disonancia" entre los derechos estudiados y los derechos ejercidos. La escuela conserva un discurso universalizante constitutivo de "lo público" que, aunque no logrado, parece mantenerse vigente como horizonte utópico (2013, p. 4).

Es el ejercicio pedagógico un escenario desde el cual se puede criticar y repensar la producción de conocimientos, identidades, estereotipos, prácticas que frente a las sexualidades se construyen. Así, se trata de reconocer en palabras de Felliti que 
la sexualidad, más que decir de nosotros mismos, habla de la cultura en la que vivimos. Los significados que le atribuimos están socialmente organizados y sostenidos por diversos lenguajes que intentan decirnos lo que es el sexo, lo que debería y lo que podría llegar a ser (2013). De esta manera, es importante que en nuestro papel de docentes hagamos un reconocimiento de las limitaciones que se nos presentan en el ejercicio de acercarnos limpia e inclusivamente a la diversidad, de reconocer cuándo, sin darnos cuenta, emergen nuestras comprensiones de mundo que responden a los estereotipos tradicionales, patriarcales y heteronormativos. Es importante mirar e interpretar de forma menos rígida las agencias de las y los estudiantes en las que se vislumbran, fugas, rupturas, resistencias, posibilidades de autocreación que abren paso a otras comprensiones del género, del sexo, de las identidades, de lo intercultural y de las sexualidades que se encuentran en el escenario académico.

Es necesario que todas y todos los encargados de educar en las escuelas lo hagan con una perspectiva de orientación como promotoras y promotores de la formación en sexualidades, con adecuadas estrategias didácticas, no solo como informadores o sancionadores (Testa, Nuñez, Ruiz y Senior, 2002).

Para Morgade (2013), las y los estudiantes aprenden cosas sobre ellos de forma implícita o explícita, en las interacciones que sostienen no solo con los textos, con los manuales, sino también con las y los docentes y compañeras (os). Estos aprendizajes les van mostrando cuál es su lugar en la sociedad que dependiendo de dichas interacciones se pueden presentar como de marginación y exclusión, o por el contrario de aceptación respeto e inclusión.

A decir de Altmann (2013), el tema de la diversidad sexual y de género debe ser incluido en el currículo de formación, no como un taller aislado, sino que debe estar presente en todas las asignaturas. De este modo, las y los docentes sensibles a estos temas pueden desarrollar estrategias de resistencia a currículos heteronormativos.

Para Morgade, Ramos, Román y Zattara (2011c), la docencia es ejercida por "intelectuales públicos". Así, con una lectura crítica de la realidad es posible desarrollar a futuro prácticas pedagógicas que apunten a construir relaciones sociales democráticas, en las que las experiencias de docentes y estudiantes no se excluyan ni silencien, sino que se constituyan en fuentes válidas de conocimiento (p. 66). 


\section{Consideraciones Finales. Interculturalidad y sexualidad: un camino para construir y recorrer}

Latinoamérica y el mundo en general en las últimas décadas han atravesado cambios de orden social, político, religioso, educativo y cultural que han movilizado otras formas de comprender aquellas realidades que aparecían inmutables; en el medio de estas transformaciones han emergido o se han visibilizado comprensiones otras relacionadas con las sexualidades. En ocasiones, la escuela pareciera rezagada de este proceso y con ella las y los diversos agentes educativos que la constituyen. Así, como se mencionó a lo largo del presente escrito, desde la perspectiva intercultural es imperante que los escenarios educativos se constituyan en espacios de apertura en los cuales no solo converjan docentes, estudiantes y eventualmente (cuando una situación crítica así lo requiere) padres de familia, sino que haya espacio para la comunidad en general, donde la práctica educativa esté permeada por la participación de todas y todos, con espacios para la construcción colaborativa del currículo, para la participación democrática y crítica en la que todos y cada uno sienta que tiene un lugar de reconocimiento y respeto.

Así, se requiere que temáticas como la diversidad y la diferencia no sean abordadas tangencialmente, sino que, por el contrario, se constituyan en ejes articuladores que inviten desde diversas expresiones: éticas, estéticas, políticas, a la discusión, al debate, a la toma de postura enmarcada en los aportes que el encuentro intercultural en el espacio académico posibilita, para ello es necesario acoger en las escuela no solo los saberes legitimados y científicos, sino saberes otros que tradicionalmente se han leído como subordinados o marginales.

De igual forma la escuela y con ella sus actores deben permitirse superar los cerramientos que la arquitectura les impone, deben acceder a otras realidades más inmediatas, dotarse de los insumos que los contextos que la rodean les ofrece, así, nociones como raza, sexo, etnia, sexualidad, diversidad, género, entre otras, que en principio son aprendidas a través de los textos, son puestas en discusión con los parámetros de la realidad.

Por último, se trata indagar en cada escenario educativo acerca de las comprensiones que circulan frente a la interculturalidad, cómo se gestionan los vacíos que frente a ella se poseen y cómo desde el trabajo inicialmente de docentes se convoca a la comunidad educativa y a la sociedad en general, para que la interculturalidad sea la base de la práctica educativa en general. 
El reto está en reconocer las sexualidades como un valor de los seres humanos. La sexualidad no debe verse como un escenario de disputa y exclusión, sino como un espacio de aprendizaje y creación, para ello, necesariamente, debe atravesar nuestra intimidad y nuestro cuerpo nos debe posibilitar un re-conocimiento de nuestros miedos, ausencias, resistencias, goces, deseos y desconocimientos. La sexualidad es mutable, no es estática y no significa que solo las y los adolescentes, las y los jóvenes deben aprender de ella, todos en nuestra humanidad debemos darle un lugar para pensarla, repensarla y de ser posible reaprenderla.

\section{Referencias}

Altmann, Helena. (2013:) Diversidade sexual e educação: desafios para a formação docente. Sexualidad, Salud y Sociedad - Revista Latinoamericana, (13), 69-82. Centro LatinoAmericano em Sexualidade e Direitos Humanos Río de Janeiro, Brasil. Recueperado de http://www.redalyc.org/articulo.oa?id=293325757003

Bruner, Jerome. (1994). Actos de significado: más allá de la revolución cognitiva. Madrid: Alianza.

Caricote, Esther. (2008). Los docentes y la educación sexual en la adolescencia. Revista Ciencias de la Educación, 18(32). Recuperado de http://servicio.bc.uc.edu.ve/educacion/revista/

Colombia, Ministerio de Educación Nacional. (2 de julio, 1993). Resolución 3353, Proyecto Nacional de Educación Sexual (PNES). Bogotá, Colombia: Diario Oficial de Colombia.

Colombia, Ministerio de Educación Nacional. (3 de agosto, 1994). Decreto 1860. Bogotá, Colombia. Recuperado de http://www.mineducacion.gov.co/1621/articles172061 archivo pdf decreto1860 94.pdf

Colombia. (1991). Constitución Política de Colombia. Bogotá: Legis.

Colombia, Ministerio de Educación Nacional. (8 de febrero, 1994). Ley General de Educación $0115 . \quad$ Bogotá, Colombia. Recuperado de http://www.mineducacion.gov.co/1621/articles-85906 archivo pdf.pdf

Colombia, Ministerio de Educación Nacional. (2003). Política Nacional de Salud Sexual y Reproductiva. Bogotá, Colombia. Recuperado de http://www.mineducacion.gov.co/1621/articles-172061 archivo pdf politicaSSR.pdf

De Aquino, Eduardo. (1999). Educação e racismo. Os educadores diante do preconceito racial. Trabalho para Discussão. Recife: Fundação Joaquim Nabuco, n. 88. Recuperado de http://www.fundaj.gov.br/geral/educacao foco/eduardoaquino.pdf 
Elizalde, Silvia. (2013). Pedagogías de la sexualidad, educación y desafíos políticos. En Género y sexualidades: debates y herramientas para una educación intercultural (pp.224). Argentina: Centro REDES.

Felliti, Karina. (2013). Construcciones históricas sobre el género y las sexualidades. Regulaciones, negociaciones y resistencias. En Género y sexualidades: debates y herramientas para una educación intercultural (pp. 26-42). Argentina: Centro REDES.

Ferreira, Sheila. (2012). O "cotidiano" e a "crítica": uma análise do preconceito sob dois posicionamentos teóricos. Psicología Revista, 21(1), 45-58. Recuperado de http://revistas.pucsp.br/index.php/psicorevista/article/view/13582/10089

Flores, Valeria (2007). Educacion sexual. Ruptura o estabilidad del contrato heterosexual. Artículos de Ciudad Mujeres. Recuperado de http://www.ciudaddemujeres.com/articulos/ Valeria-Flores

Heller, Agnes. (1991). Sociología de la vida cotidiana. Barcelona: Península.

Leiva Olivencia, Juan. (2010). La educación intercultural entre el deseo y la realidad: reflexiones para la construcción de una cultura de la diversidad en la escuela inclusiva. Revista Docencia e Investigación, 10(20), 149-182. Recuperado de http://www.uclm.es/varios/revistas/docenciaeinvestigacion/pdf/numero10/6.pdf

Lopes Louro, Guacira. (2000). O corpo educado pedagogias da sexualidade. Belo Horizonte, Brasil: Autêntica.

Martínez, Luz. (2012). La Educacion Intercultural desde la perspectiva de género. Interculturalidad y práctica docente. España: Red de Escuelas Interculturales.

Morgade, Graciela. (2013). Educación sexuada y curriculum: Debates Epistemológicos y didácticos actuales. En Género y sexualidades: debates y herramientas para una educación intercultural (pp. 01-16). Argentina: Centro REDES.

Morgade, Graciela (Coord.). (2011). Toda educación es sexual. Hacia una educación sexuada justa. Buenos Aires: La Crujía.

Morgade, Graciela. (2006). Educación en la sexualidad desde el enfoque de género. Una antigua deuda en la escuela. Buenos Aires: Novedades Educativas.

Morgade, Graciela, Baez, Jesica, Zattara, Susana y Díaz, Gabi. (2011). Pedagogías, teorías de género y tradiciones en "educación sexual". En Graciela Morgade (Coord.), Toda educación es sexual. Hacia una educación sexuada justa (pp. 22-51). Buenos Aires: La Crujía.

Morgade, Graciela, Ramos, Gabriela, Román, Cecilia y Zattara, Susana. (2011). Visiones de directivos/as docentes. En Graciela Morgade (Coord.), Toda educación es sexual Hacia una educación sexuada justa (pp. 53-80). Buenos Aires: La Crujía.

Muñoz, Germán. (2012). Youth studies in Colombia: State of the Art. En Colombia Young Ed. Sage Publications. (pp.399-414). Colombia: District University of Bogotá. 
PROFAMILIA. (2010). Encuesta Nacional en Demografía y Salud-ENDS. Colombia. Recuperado

de http://www.profamilia.org.co/encuestas/Profamilia/Profamilia/index.php?option=com co $\underline{\text { ntent } \& \text { view }=\text { article\&id }=62 \& \text { Itemid }=9}$

Secretaría de Educación Distrital, Fundación Gamma Idear. (2006). Caracterización de los proyectos en educación sexual y prevención en los colegios distritales. Bogotá: Secretaria de Educación Distrital. Recuperado de: https://centrodocumentacion.educacionbogota.edu.co/jspui/handle/123456789/53

Valencia Jiménez, Nydia Ninna y Solera Martínez, Néstor Fidel. (2011) Necesidades de aprendizaje para la sexualidad de jóvenes entre 10 y 19 años en el Caribe colombiano. Investigación y Desarrollo, 17(1), 106-131. Recuperado de http://ciruelo.uninorte.edu.co/pdf/invest desarrollo/171/05\%20NECESIDADES\%20DE\%20APRENDIZAJE.pdf

Testa, Mirian, Nuñez, María, Ruiz, Fredy y Senior, Alexa. (2002). Nivel de educación sexual de los docentes. Revista Multiciencias, 2(2), 107-114. Recuperado de http://www.redalyc.org/articulo.oa?id=90420204

Torres, Yolanda, Berbesi, Dedsy, Bareño, José y Montoya, Liliana. (2010). Situación de salud mental del adolescente en Colombia. Estudio nacional de salud mental Colombia, 2003. Medellín: Universidad CES. 